\section{Aortic Dissection in the Setting of Concomitant Use of MAOI and Trazodone: A Case Report}

\author{
Thomas J Blount, Varun Monga* Kellie Nelson and Ashish \\ Sharma
}

Department of Psychiatry, University of Nebraska Medicine Center, Omaha, Nebraska, USA

\begin{abstract}
The use of Monoamine Oxidase Inhibitor (MAOI) medications in the treatment of depression has largely fallen out of favor due to the well-chronicled association with hypertensive crisis and resultant morbidity. Hypertensive crises have been noted to cause several manifestations of end-organ damage such as sub-arachnoid hemorrhage or even death. To our knowledge, however, there is no documented case which implicates MAOI use and subsequent hypertensive crisis in the etiology of aortic dissection. We present here what we believe to be a potentially novel example of end-organ damage in the context of MAOI use as a possible contributing factor.
\end{abstract}

\section{Introduction}

The topic of hypertensive crisis in the context of MAOI treatment has been studied extensively, ever since the association was first noted in 1964 [1]. The prescribing of MAOI antidepressants has largely fallen out of favor due to the complex and largely dangerous side effect profile of this drug class. The most feared complication of MAOI use is hypertensive crisis. These crises often lead to end organ damage as severe as subarachnoid hemorrhage or death. We present here what we believe to be a possibly novel presentation of end organ damage during a potential hypertensive crisis in a middle-aged female taking phenelzine.

\section{Case Report}

We describe the case of a 51-year-old female depressed patient whom we came to know during her hospital stay. She was previously unknown to our psychiatry practice, but had received psychiatric care at an outside facility for many years prior to the present episode. She had no history of drug use, and no pre-existing medical diagnoses, besides her depression and anxiety. Of note, she had never had blood

*Corresponding author: Varun Monga, Department of Psychiatry, University of Nebraska Medicine Center, Omaha, Nebraska, USA, Tel: +1 4025171315 ; Fax: +1 4025526247; E-mail: monga.varun81@gmail.com

Citation: Blount TJ, Monga V, Nelson K, Sharma A (2015) Aortic Dissection in the Setting of Concomitant Use of MAOI and Trazodone: A Case Report. J Clin Stud Med Case Rep 2: 017.

Received: April 10, 2015; Accepted: June 06, 2015; Published: June 24, 2015 pressure readings outside the normal range. A preoperative lipid panel revealed values which were all in the normal range, and an HDL that was 63 , in the "optimal" range. Additionally, she had no personal or family history of heart disease, aortic dissection, aortic valvular disease, or connective tissue disease.

Over the years, she had attempted trials of many antidepressants belonging to several drug classes, including several SSRI's (Selective Serotonin Reuptake Inhibitors) and at least one SSNRI (Selective Serotonin and Norepinephrine Reuptake Inhibitor). Her depression was refractory to all these treatments, so her physician started her on phenelzine. Her dose was titrated to $45 \mathrm{mg}$ per day in split doses, 30 $\mathrm{mg}$ in the morning and $15 \mathrm{mg}$ at night. Aside from her depression, she also dealt with anxiety and insomnia, for which she took gabapentin $100 \mathrm{mg}$ twice daily and trazodone $50 \mathrm{mg}$ at bedtime, respectively. Two weeks before her presentation, she felt that her depression was worsening, and she was instructed to increase her dose of phenelzine to $60 \mathrm{mg}$ per day: $30 \mathrm{mg}$ twice a day. Two weeks after increasing her dose, she began to have chest pains.

Our patient presented to the hospital ER with the classic signs and symptoms of an aortic dissection: abrupt onset of sharp, tearing, substernal chest pain which began just after she ate dinner. A chest CT obtained in the ER revealed a Stanford Type A Aortic Dissection involving her carotid arteries bilaterally. At presentation she was afebrile at $36.7^{\circ} \mathrm{C}$, and her blood pressure was $94 / 61$ after she was started on a nitroglycerin drip en route to the hospital. She was sent to the OR for emergent repair of her dissection. Intraoperative specimens were sent to pathology, and revealed "patchy elastin loss and associated increased acid mucopolysaccharide-rich ground substance," and an echocardiogram showed mild, Katz grade II/V intimal thickening. Unfortunately our patient's medical recovery was complicated by a post-operative embolic stroke, which resulted in hemiparesis and diplopia. Her medical condition improved over the period of several weeks, and her psychiatric recovery is ongoing, as is the investigation into the factors that may have lead to the aortic dissection.

\section{Discussion}

The combination of Trazodone and Phenelzine is listed as a severe drug-drug interaction, with the advertised possibility of serotonin syndrome [2]. This syndrome is usually characterized by the development of hyperthermia, hypertension, autonomic instability, and changes in mental status, among other effects.

The timing of this aortic dissection, having occurred just two weeks after a recent increase in phenelzine dosage, is significant in considering the combination of our patient's medications and hypertensive crisis as a possible etiology of the event that lead to her aortic dissection. Pathology did reveal pre-existing mild aortic disease in our patient, so it is plausible to consider acute hypertension as a contributing factor which pushed her aorta past its breaking point. Nowhere in the current literature is there reference to aortic dissection resultant from hypertensive crisis caused by MAOI use. There are examples of myocardial damage and myonecrosis attributable to phenelzine or other MAOI's [3,4], but no specific mention of aortic dissection. A prospective cohort study reported that 
hypertension and smoking are the top two associated conditions in those who suffer aortic dissection. It was found that $86 \%$ of patients who suffered aortic dissection had a documented history of hypertension. Hypertension contributed $54 \%$ of the Population-Attributable Risk (PAR) for aortic dissection, and smoking contributed $14 \%$ of the PAR. Another significant chunk of the PAR came from low levels of apolipoprotein A1, which is a protein that comprises HDL. These 3 factors combined to account for $98 \%$ of the PAR for aortic dissection, as determined by the study parameters [5]. Only one study found any reference whatsoever to aortic dissection in the context of MAOI use. This study examined the effect of chemically-induced aortic rupture in turkeys, and the protective or enhancing properties of MAOI's and reserpine. One turkey cohort was treated with MAOI's, and the other was treated with reserpine, an antipsychotic with antihypertensive properties. Both cohorts, along with a control group, were given Beta-Aminopropionitrile (BAPN), a compound shown to cause aortic dissection and rupture in turkeys. The results showed that MAOI use potentiated and enhanced the effects of BAPN, and caused a significant increase in the incidence and severity of aortic dissection [6].

We believe this present case to be a potentially novel presentation of aortic dissection in the context of dangerous pharmacological combinations. We cannot claim to know exactly what caused this dissection, since we do not know what her blood pressure was immediately before the event. We also acknowledge that is entirely plausible that this dissection occurred randomly as a direct result of intimal disease and bad luck. Our subject is a non-smoker with no documented history of either drug use or hypertension, along with an optimal HDL level, and no apparent major risk factors that would predispose her to suffering an aortic dissection. Thus, given the timing of her medication changes and the well-documented potential for hypertensive crisis with MAOI use, we cannot rule out medication-induced hypertensive crisis as a possible contributing factor in the case of this patient's aortic dissection.

\section{References}

1. Bethune HC, Burrell RH, Culpan RH, Ogg GJ (1964) Vascular crises associated with monoamine-oxidase inhibitors. Am J Psychiatry 121: 245-248.

2. Ritter JL, Alexander B (1997) Retrospective study of selegiline-antidepressant drug interactions and a review of the literature. Ann Clin Psychiatry 9: $7-13$

3. Ngo AS, Ho RY, Olson KR (2010) Phenelzine-induced myocardial injury: a case report. J Med Toxicol 6: 431-434.

4. Liu LX, Rustgi AK (1987) Cardiac myonecrosis in hypertensive crisis associated with monoamine oxidase inhibitor therapy. Am J Med 82: 1060-1064.

5. Landenhed M, Engström G, Gottsäter A, Caulfield MP, Hedblad B, et al. (2015) Risk profiles for aortic dissection and ruptured or surgically treated aneurysms: a prospective cohort study. J Am Heart Assoc 4: 001513.

6. Simpson CF, Kling JM, Robbins RC, Harms RH (1968) Beta-Aminopropionitrile-induced aortic ruptures in turkeys: inhibition by reserpine and enhancement by monoamine oxidase inhibitors. Toxicol Appl Pharmacol 12: 48-59. 